\title{
A Shrinking Projection Algorithm with Errors for Costerro Bounded Linear Mappings
}

\author{
Joseph Frank Gordon \\ Department of Mathematics, Nanjing University of Aeronautics and Astronautics, Nanjing 211106, China \\ Correspondence should be addressed to Joseph Frank Gordon; jgordon@aims.edu.gh
}

Received 9 January 2020; Revised 25 March 2020; Accepted 31 March 2020; Published 29 April 2020

Academic Editor: Tepper L Gill

Copyright () 2020 Joseph Frank Gordon. This is an open access article distributed under the Creative Commons Attribution License, which permits unrestricted use, distribution, and reproduction in any medium, provided the original work is properly cited.

\begin{abstract}
The purpose of this paper is to introduce and analyze the shrinking projection algorithm with errors for a finite set of costerro bounded linear mappings in the setting of uniformly convex smooth Banach spaces. Here, under finite dimensional or compactness restriction or the error term being zero, the strong limit point of the sequence stated in the iterative scheme for these mappings in uniformly convex smooth Banach spaces was studied. This paper extends Ezearn and Prempeh's result for nonexpansive mappings in real Hilbert spaces.
\end{abstract}

\section{Introduction}

Fixed-point theory is a fascinating subject, with a lot of applications in various fields of mathematics and engineering. In a number of situations, one may need to find a common fixed point of a family of mappings. In practice, a modification may be needed to turn the problem into a fixed-point problem (see, for instance, Picard [1] and Lindelöf [2]). For more information on the fixed-point problem and its applications to certain types of linear and nonlinear problems, interested readers should be referred to Tang and Chang [3] (equilibrium problems), Solodov and Svaiter [4] (proximal point algorithm), Takahashi [5, 6] (convex optimization and minimization problems), and Blum and Oettli [7] (variational inequalities).

In practice, finding an exact closed form of a solution to a fixed-point problem is almost a difficult task. For this reason, it has been of particular importance in the development of feasible iterative schemes or methods for approximating fixed points of certain maps, most notably, nonexpansive type of mappings. For instance, Halpern [8], Mann [9], and Ishikawa [10] studied and developed an iterative scheme to approximate the fixed points of nonexpansive mappings in Hilbert spaces under certain conditions. In their scheme, strong convergence is always guaranteed for all closed convex subsets of a Hilbert space. Haugazeau [11] initially proposed the projection method which was later developed by Solodov and Svaiter [4]. A type of projection method which is of relevance and central to this paper is called the Shrinking Projection Method with Errors, which was developed by Takahashi et al. [12] and used by Yasunori [13]. Strong convergence result is always guaranteed for all closed convex subsets of a Hilbert space under certain conditions.

In [14], Ezearn and Prempeh improved the boundedness requirement of Yasunori's result [13] regarding a shrinking projection algorithm for common fixed points of nonexpansive mappings in a real Hilbert space. In their results, they showed that the boundedness requirement in Yasunori's results could be removed. That is to say that the convergence of the iterative sequence in the scheme presented in Yasunori's paper, that is, the error term $\varepsilon_{0}=0$ is independent of the boundedness of the closed convex subset in a real Hilbert space. With the boundedness removed, Ezearn and Prempeh further provided a better estimate for the convergence result of the iterative sequence in their algorithm especially in finite dimensional and further showed that when the closed convex set is compact, their estimates do not involve the diameter of the subset.

In this paper, it is shown that the strong limit point of the iterative sequence $\left\{x_{n}\right\}_{n \geq 1}$ presented in Iterative Scheme 1 
always exists in a finite-dimensional space. And, it also shown that when the space is not finite dimensional, the strong limit point of $\left\{x_{n}\right\}_{n \geq 1}$ is guaranteed when the closed convex subset is compact. Finally, the strong limit point of $\left\{x_{n}\right\}_{n \geq 1}$ also exists when the error term $\left(\varepsilon_{0}\right)$ is zero regardless of the compactness of the closed convex subset and the dimension of the space.

Definition 1 (normalised duality mapping, see Lunner [15]). Let $\mathscr{X}$ be a Banach space with the norm $\|\cdot\|$ and let $\mathscr{X}^{*}$ be the dual space of $\mathscr{X}$. Denote $\langle\cdot, \cdot\rangle$ as the duality product. The normalised duality mapping $J$ from $\mathscr{X}$ to $\mathscr{X}^{*}$ is defined by

$$
J x:=\left\{f \in \mathscr{X}^{*}:\|f\|_{*}^{2}=\|x\|^{2}=\langle x, f\rangle=f x\right\},
$$

for all $x \in \mathscr{X}$. The Hahn Banach theorem guarantees that $J x \neq \varnothing$ for every $x \in \mathscr{X}$. For the purposes of this paper, the interest mostly lies on the case when $J x$ is single valued for all $x \in \mathscr{X}$, which is equivalent to the statement that $\mathscr{X}$ is a smooth Banach space.

Throughout this paper, $\mathfrak{R}$ denotes the real part of a complex number and $F(T)$ is used to denote the set of fixed points of the mapping $T$ (that is, $F(T)=\{x \in \mathscr{C}: T x=x\}$ ).

The mappings studied in this paper are defined in the following.

Definition 2 (costerro bounded linear mappings). Let $\mathscr{X}$ be a strictly convex smooth reflexive space and $\mathscr{C}$ a closed convex subset of $\mathscr{X}$. A mapping $T: \mathscr{C} \longrightarrow \mathscr{X}$ is said to be a costerro bounded linear mapping if

$$
\|T x\| \leq\|x\|,
$$

such that whenever $z \in F(T)$, then

$$
\mathfrak{R}\langle z, J T x-J x\rangle \geq 0, \quad \forall x, z \in \mathscr{C} .
$$

An immediate example of such mappings is the scaling operator given by

$$
T(x)=a x,
$$

where the scaling factor $a$ lies in the closed unit disk.

In order to state the iterative scheme, the following function is defined.

Definition 3 (generalised projection functional, see Alber [16]). Let $\mathscr{X}$ be a smooth Banach space and let $\mathscr{X}^{*}$ be the dual space of $\mathscr{X}$. The generalised projection functional $\phi(\cdot, \cdot): \mathscr{X} \times \mathscr{X} \longrightarrow \mathbb{R}$ is defined by

$$
\phi(y, x)=\|y\|^{2}-2 \Re\langle y, J x\rangle+\|x\|^{2},
$$

for all $x, y \in \mathscr{X}$, where $J$ is the normalised duality mapping from $\mathscr{X}$ to $\mathscr{X}^{*}$. It is obvious from the definition that the generalised projection functional $\phi(\cdot, \cdot)$ satisfies the following inequality:

$$
(\|y\|-\|x\|)^{2} \leq \phi(y, x) \leq(\|y\|+\|x\|)^{2},
$$

for all $x, y \in \mathscr{X}$.

Note that the generalised projection functional $\phi(\cdot, \cdot)$ is continuous.
The next function which is stated in the iterative scheme is established via the following theorem .

Theorem 1 (generalised projection, see Li [17]). Let $\mathscr{X}$ be a uniformly convex smooth Banach space and let $\mathscr{C} \neq \varnothing$ be a closed convex subset of $\mathscr{X}$. Then, for every $x \in \mathscr{X}$, there exists $a$ unique $y \in \mathscr{C}$ such that

$$
\Lambda(x, C)=\phi(y, x)=\inf _{z \in \mathscr{C}} \phi(z, x) .
$$

The unique point $y$ satisfying equation (7) is the called the generalised projection of $x$ on $\mathscr{C}$. That is, the projection operator $\Pi_{\mathscr{C}}: \mathscr{X} \longrightarrow \mathscr{C}$ is defined by setting

$$
\Pi_{\mathscr{C}} x=y
$$

where $y$ is the only point in $\mathscr{C}$ satisfying equation (7).

Remark 1. In Theorem 1 , note that if $\mathscr{X}$ is a Hilbert space, then $\phi(y, x)=\|y-x\|^{2}$. Hence, the (generalised) projection $\Pi_{\mathscr{C}}$ defined in equation (8) coincides with the metric projection onto $\mathscr{C}$ in the Hilbert space setting. The converse is not necessarily true in a general Banach space.

The iterative scheme is stated as follows.

Iterative Scheme 1 . Let $\mathscr{X}$ be a uniformly convex smooth Banach space and let $\mathscr{C} \neq \varnothing$ (not necessarily bounded) be a closed convex subset of $\mathscr{X}$. Let $\left\{T_{k}\right\}_{k=1}^{m}$ be finite set of costerro bounded linear mappings from $\mathscr{C}$ to $\mathscr{X}$ with $F:=\cap_{k=1}^{m} F\left(T_{k}\right) \neq \varnothing$. Let $\left\{\alpha_{n, k}\right\}_{n \geq 1}$ and $\left\{\varepsilon_{n}\right\}_{n \geq 1}$ be nonnegative real sequences satisfying the following conditions:

(i) $\left\{\alpha_{n, k}\right\}_{n \geq 1} \subset[0,1]$

(ii) $\sum_{k=1}^{m} \alpha_{n, k}=1$

(iii) $\alpha_{k}:=\liminf _{n \longrightarrow \infty} \alpha_{n, k}>0$

(iv) $\varepsilon_{0}:=\lim \sup _{\mathrm{n} \longrightarrow \infty} \varepsilon_{n}<\infty$

for all $1 \leq k \leq m$ and $n \geq 1$.

Then, for any arbitrary $u \in \mathscr{X}$ with the assumptions $x_{1} \in \mathscr{C}_{1}:=\mathscr{C}$ and $\phi\left(x_{1}, u\right)<\varepsilon_{1}^{2}$, the sequence $\left\{x_{n}\right\}_{n \geq 1}$ is defined iteratively by the following scheme:

$$
\begin{aligned}
\mathscr{B}_{n} & =\left\{z \in \mathscr{C}: \sum_{k=1}^{m} \alpha_{n, k} \phi\left(z, T_{k} x_{n}\right) \leq \phi\left(z, x_{n}\right)\right\}, \\
\mathscr{C}_{n+1} & =\mathscr{B}_{n} \cap \mathscr{C}_{n}, x_{n+1} \in \mathscr{C}_{n+1}, \\
\phi\left(x_{n+1}, u\right) & \leq \Lambda\left(u, \mathscr{C}_{n+1}\right)+\varepsilon_{n+1}^{2},
\end{aligned}
$$

for all $n \geq 1$.

\section{Preliminaries}

The inequality $\mathfrak{R}\langle z$,JT $x-J x\rangle \geq 0$ in Definition 2 can be written equivalently in terms of norms. This is achieved via the elementary lemma by Ezearn in [18]. The proof is given here for the sake of completeness. 
Theorem 2 (see, for instance, Ezearn [18]). Let $X$ be a smooth Banach space and let $x \in \mathscr{X} \backslash\{0\}$ and any $y \in \mathscr{X}$. Then,

$$
\frac{1}{\|x\|} \mathfrak{R}\langle y, J x\rangle=\lim _{\alpha \longrightarrow 0} \frac{\|x+\alpha y\|-\|x\|}{\alpha},
$$

for all $\alpha>0$.

Lemma 1 (see Ezearn [18]). Let $\mathscr{X}$ be a smooth Banach space and $x_{1}, x_{2}, \ldots, x_{m}, y \in \mathscr{X}$ where $m \in \mathbb{N}$. Then,

$$
\sum_{k=1}^{m}\left\|x_{k}\right\|^{2} \leq \sum_{k=1}^{m}\left\|x_{k}\right\|\left\|x_{k}+\alpha y\right\|
$$

for all $\alpha \in[0, q]$ (where $q \in \mathbb{R}_{>0}$ ) if and only if

$$
\mathfrak{R}\left\langle y, J x_{1}+J x_{2}+\cdots+J x_{m}\right\rangle \geq 0 \text {. }
$$

Proof. If $\alpha=0$, then the lemma is proved trivially, and as a result, it is assumed that $\alpha \neq 0$ (without loss of generality, it is equally assumed that $\left.x_{k} \neq 0\right)$. Now, if $\mathfrak{R} \sum_{k=1}^{m}\left\langle y, J x_{k}\right\rangle \geq 0$, then

$$
\begin{aligned}
\sum_{k=1}^{m}\left\|x_{k}\right\|^{2} & =\Re \sum_{k=1}^{m}\left\langle x_{k}, J x_{k}\right\rangle \leq \mathfrak{R} \sum_{k=1}^{m}\left\langle x_{k}+\alpha y, J x_{k}\right\rangle \\
& \leq \sum_{k=1}^{m}\left\|x_{k}+\alpha y\right\|\left\|x_{k}\right\| .
\end{aligned}
$$

On the contrary, if $\sum_{k=1}^{m}\left\|x_{k}\right\|^{2} \leq \sum_{k=1}^{m}\left\|x_{k}\right\|\left\|x_{k}+\alpha y\right\|$ for every $\alpha \in(0, q]$ (where $q \in \mathbb{R}_{>0}$ ), then

$$
0 \leq \frac{1}{\alpha} \sum_{k=1}^{m}\left\|x_{k}\right\|\left(\left\|x_{k}+\alpha y\right\|-\left\|x_{k}\right\|\right)=\sum_{k=1}^{m}\left\|x_{k}\right\| \frac{\left\|x_{k}+\alpha y\right\|-\left\|x_{k}\right\|}{\alpha} .
$$

Taking the limit as $\alpha \longrightarrow 0$, then by Theorem 2 , equation (14) becomes

$$
\frac{1}{\left\|x_{k}\right\|} \Re \sum_{k=1}^{m}\left\langle y, J x_{k}\right\rangle \geq 0 .
$$

Since $x_{k} \neq 0$, then $\mathfrak{R} \sum_{k=1}^{m}\left\langle y, J x_{k}\right\rangle \geq 0$ and hence proved.

Corollary 1. The inequality $\Re\langle z, J T x-J x\rangle \geq 0$ is equivalent to

$$
\|T x\|^{2}+\|x\|^{2} \leq\|T x\|\|T x+\alpha z\|+\|x\|\|x-\alpha z\|,
$$

for all $\alpha \geq 0$.

Proof. By considering Lemma 1 for the case when $m=2$, the inequality

$$
\mathfrak{R}\left\langle z, J x_{1}-J x_{2}\right\rangle=\mathfrak{R}\left\langle z, J x_{1}+J\left(-x_{2}\right)\right\rangle \geq 0,
$$

is equivalent to the following condition:

$$
\begin{gathered}
\sum_{k=1}^{2}\left\|x_{k}\right\|^{2} \leq \sum_{k=1}^{2}\left\|x_{k}\right\|\left\|x_{k}+\alpha y\right\|, \\
\left\|x_{1}\right\|^{2}+\left\|x_{2}\right\|^{2} \leq\left\|x_{1}\right\|\left\|x_{1}+\alpha y\right\|+\left\|x_{2}\right\|\left\|x_{2}+\alpha y\right\| .
\end{gathered}
$$

Now, replacing $x_{1}$ with $T x, y$ with $z$, and $x_{2}$ with $(-x)$, the corollary is proved.

Below, a nontrivial example of costerro bounded linear mappings is given which is referred to as Ezearn nonexpansive mapping. Ezearn, in his thesis [18], had defined certain closely related mappings (named type III variational nonexpansive mappings).

Corollary 2 (Ezearn nonexpansive mapping). Let $\mathscr{C}$ be a closed convex subset of a strictly convex smooth reflexive space $\mathscr{X}$. Then, the following is a nontrivial example of a costerro bounded linear mapping:

$$
\begin{aligned}
& \left(\|T x\|^{2}+\|T y\|^{2}\right)+\left(\|x\|^{2}-\|y\|^{2}\right) \\
& \quad \leq\|T x\|\|T x+\alpha T y\|+\|x\|\|x-\alpha y\|,
\end{aligned}
$$

for all $x, y \in \mathscr{C}$ and all $\alpha \geq 0$.

Proof. For $\alpha=0$, equation (19) reduces to the following:

$$
\begin{gathered}
\|T x\|^{2}+\|T y\|^{2}+\|x\|^{2}-\|y\|^{2} \leq\|T x\|^{2}+\|x\|^{2}, \\
\|T y\|^{2} \leq\|y\|^{2}, \\
\|T y\| \leq\|y\|,
\end{gathered}
$$

which satisfies the first part of Definition 2. To show the second part of Definition 2, if $y \in F(T)$, where $F(T)$ refers to the fixed point set of $T$, then equation (19) reduces to the following evaluation:

$$
\begin{array}{r}
\left(\|T x\|^{2}+\|y\|^{2}\right)+\left(\|x\|^{2}-\|y\|^{2}\right) \leq\|T x\|\|T x+\alpha y\|+\|x\|\|x-\alpha y\|, \\
\|T x\|^{2}+\|x\|^{2} \leq\|T x\|\|T x+\alpha y\|+\|x\|\|x-\alpha y\|,
\end{array}
$$

which by Corollary 1 is equivalent to $\mathfrak{R}\langle y, J T x-J x\rangle \geq 0$. Hence proved.

Lemma 2 (see, for instance, Ezearn [18]). Let $\left\{\mathscr{C}_{n}\right\}_{n \geq 1}$ be a sequence of nonempty closed convex subsets of a uniformly convex smooth Banach space $\mathscr{X}$ such that $\mathscr{C}_{n+1} \subset \mathscr{C}_{n}$. Suppose that further that $\mathscr{C}_{\infty}=\cap_{n \geq 1} \mathscr{C}_{n}$ is nonempty. Then, the sequence of generalized projections $\left\{\Pi_{\mathscr{C}_{n}} x\right\}_{n \geq 1}$ converges strongly to $\Pi_{\mathscr{C}_{\infty}} x$ for any $x \in \mathscr{X}$.

Proposition 1 (seeAlber [19], Alber and Reich [20], and Kamimura and Takahashi [21]). Let $\mathscr{X}$ be a real uniformly convex smooth Banach space and $\mathscr{C} \neq \varnothing$ be a closed convex subset of $\mathscr{X}$. Then, the following inequality holds:

$$
\phi\left(y, \Pi_{\mathscr{C}} x\right)+\phi\left(\Pi_{\mathscr{C}} x, x\right) \leq \phi(y, x),
$$

for all $y \in \mathscr{C}$ and $x \in \mathscr{X}$.

Proposition 2 (continuity in duality pairing). Let $\mathscr{X}$ be a Banach space and let $\mathscr{X}^{*}$ be the dual space of $\mathscr{X}$. Denote $\langle\cdot, \cdot\rangle$ as the duality product. Now, for $\left\{x_{n}\right\}_{n \geq 1} \subset \mathscr{X}$ and $\left\{f_{n}\right\}_{n \geq 1} \subset \mathscr{X}^{*}$, suppose either of the following conditions hold:

(i) $\left\{x_{n}\right\} \% \rightarrow x$ and $\left\{f_{n}\right\} \longrightarrow f$

(ii) $\left\{x_{n}\right\} \% \rightarrow x$ and $\left\{f_{n}\right\} \stackrel{*}{\rightarrow} f$

Then, $\lim _{n \longrightarrow \infty}\left\langle x_{n}, f_{n}\right\rangle=\langle x, f\rangle$. 
Lemma 3 (weak star-continuity in smooth spaces). Let $\mathscr{X}$ be a real smooth Banach space. Then, $J: \mathscr{X} \longrightarrow \mathscr{X}^{*}$ is norm-toweak star continuous, where $J$ is the normalized duality mapping.

Lemma 4 (see Kamimura and Takahashi [21]). Let $\mathscr{X}$ be a uniformly convex and smooth Banach space and let $\left\{x_{n}\right\}$ and $\left\{y_{n}\right\}$ be two sequences in $\mathscr{X}$ such that either $\left\{x_{n}\right\}$ or $\left\{y_{n}\right\}$ is bounded. If $\lim _{n \longrightarrow \infty} \phi\left(x_{n}, y_{n}\right)=0$, then $\lim _{n \rightarrow \infty} \| x_{n}-$ $y_{n} \|=0$.

\section{Main Results}

The proof of the main result of this paper is given in this section, which is accomplished in Theorem 3. The following corollary and lemmas shall aid in arriving at the conclusion of the main result.

Corollary 3. If the sequence $\left\{x_{n}\right\}_{n \geq 1}$ has a strong limit point, say $x$, then $x \in F=\cap_{k=1}^{m} F\left(T_{k}\right)$.

Proof. Without loss of generality, it is assumed that the sequence $\left\{x_{n}\right\}_{n \geq 1}=x_{1}, x_{2}, x_{3}, \ldots$ is the subsequence converging to $x$. Now, for $n \geq 1$, since the sets $\mathscr{C}_{n}$ form a decreasing sequence of sets, that is, $\mathscr{C}_{n+1} \subset \mathscr{C}_{n}$, then from Iterative Scheme $1, x_{n+1} \in \mathscr{C}_{n+1} \subset \mathscr{C}_{n}$, where $\left\{x_{n+1}\right\}_{n \geq 1}=$ $x_{2}, x_{3}, x_{4}, \ldots$. Hence, it is observed that

$$
\sum_{k=1}^{m} \alpha_{n, k} \phi\left(x_{n+1}, T_{k} x_{n}\right) \leq \phi\left(x_{n+1}, x_{n}\right)
$$

Hence, taking limit as $n \longrightarrow \infty$ of the above inequality, the following is obtained:

$$
\lim _{n \longrightarrow \infty} \sum_{k=1}^{m} \alpha_{n, k} \phi\left(x_{n+1}, T_{k} x_{n}\right) \leq \lim _{n \longrightarrow \infty} \phi\left(x_{n+1}, x_{n}\right) .
$$

By Proposition 2 and Lemma 3, $\lim _{n \longrightarrow \infty} \phi\left(x_{n+1}, x_{n}\right) \longrightarrow 0$ and as a result, the following is obtained:

$$
\lim _{n \longrightarrow \infty} \sum_{k=1}^{m} \alpha_{n, k} \phi\left(x_{n+1}, T_{k} x_{n}\right) \leq 0 .
$$

Since the generalised functional $\phi(\cdot, \cdot)$ is nonnegative and the limit infimum of $\left\{\alpha_{n, k}\right\}$ is nonzero for all $k$, the following is obtained:

$$
\lim _{n \longrightarrow \infty} \phi\left(x_{n+1}, T_{k} x_{n}\right)=0,
$$

for all $k \in\{1, \ldots, m\}$.

So by Lemma 4 ,

$$
\lim _{n \longrightarrow \infty}\left\|x_{n+1}-T_{k} x_{n}\right\|=0,
$$

for all $k \in\{1, \ldots, m\}$ and that proves the corollary due to the continuity of the norm functional and the mappings $T_{k}$.

Lemma 5. For all $n \geq 1$, the sets $\mathscr{B}_{n}$ and $\mathscr{C}_{n}$ in Iterative Scheme 1 are closed convex sets.
Proof. Because $\mathscr{C}_{1}:=\mathscr{C}$ is a closed convex set by assumption, it suffices to show that $\mathscr{B}_{n}$ is a closed convex set for all $n$. To prove the closure aspect of the lemma, if $\left\{z_{j}\right\}_{j \geq 1} \subset \mathscr{B}_{n}$ converges to $z \in \mathscr{C}$, then via the continuity of the generalised functional $\phi(\cdot, \cdot)$, the following is obtained:

$$
\begin{aligned}
\sum_{k=1}^{m} \alpha_{n, k} \phi\left(z, T_{k} x_{n}\right) & =\lim _{j \longrightarrow \infty} \sum_{k=1}^{m} \alpha_{n, k} \phi\left(z_{j}, T_{k} x_{n}\right) \\
& \leq \lim _{j \longrightarrow \infty} \phi\left(z_{j}, x_{n}\right)=\phi\left(z, x_{n}\right),
\end{aligned}
$$

and as a result, $z \in \mathscr{B}_{n}$.

Finally, to prove convexity, let $u, v \in \mathscr{B}_{n}$ and $t \in[0,1]$. First, note that whenever $z \in \mathscr{B}_{n}$, then the inequality is obtained:

$$
\sum_{k=1}^{m} \alpha_{n, k} \phi\left(z, T_{k} x_{n}\right) \leq \phi\left(z, x_{n}\right)
$$

which can be expanded and observed to be equivalent to

$$
\sum_{k=1}^{m} \alpha_{n, k}\left(\left\|T_{k} x_{n}\right\|^{2}-\left\|x_{n}\right\|^{2}\right) \leq 2 \Re \sum_{k=1}^{m} \alpha_{n, k}\left\langle z, J T_{k} x_{n}-J x_{n}\right\rangle .
$$

So by making the substitution $z=u$ and multiplied by $t$ and adding it to $z:=v$ multiplied by $(1-t)$, the following is obtained:

$$
\begin{aligned}
& \sum_{k=1}^{m} \alpha_{n, k}\left(\left\|T_{k} x_{n}\right\|^{2}-\left\|x_{n}\right\|^{2}\right) \\
& =t \sum_{k=1}^{m} \alpha_{n, k}\left(\left\|T_{k} x_{n}\right\|^{2}-\left\|x_{n}\right\|^{2}\right) \\
& +(1-t) \sum_{k=1}^{m} \alpha_{n, k}\left(\left\|T_{k} x_{n}\right\|^{2}-\left\|x_{n}\right\|^{2}\right) \\
& \leq 2 t \Re \sum_{k=1}^{m} \alpha_{n, k}\left\langle u, J T_{k} x_{n}-J x_{n}\right\rangle \\
& +2(1-t) \Re \sum_{k=1}^{m} \alpha_{n, k}\left\langle v, J T_{k} x_{n}-J x_{n}\right\rangle \\
& =2 \Re \sum_{k=1}^{m} \alpha_{n, k}\left\langle t u+(1-t) v, J T_{k} x_{n}-J x_{n}\right\rangle,
\end{aligned}
$$

from which it is concluded that

$$
\sum_{k=1}^{m} \alpha_{n, k} \phi\left(t u+(1-t) v, T_{k} x_{n}\right) \leq \phi\left(t u+(1-t) v, x_{n}\right) .
$$

Hence, $\mathscr{B}_{n}$ is convex.

Now, define

$$
\mathscr{C}_{\infty}:=\bigcap_{n \geq 1} \mathscr{C}_{n}
$$

Lemma 6. The set $\mathscr{C}_{\infty}$ is a closed convex set containing $F$. Hence, the sequence $\left\{\Pi_{\mathscr{C}_{n}} x\right\}_{n \geq 1}$ of generalised projections converges strongly to $\Pi_{\mathscr{C}_{\infty}} x$ for any arbitrary $x$ in a uniformly convex smooth Banach space $\mathscr{X}$.

Proof. By induction, it is observed that the sets $\mathscr{C}_{n}$ are all closed convex subsets by the help of Lemma 5 and the 
definition of $\mathscr{C}_{n+1}$ in Iterative Scheme 1. Moreover, by inclusion, these sets $\mathscr{C}_{n}$ form a decreasing sequence of sets. That is, $\mathscr{C}_{n+1} \subset \mathscr{C}_{n}$ for all $n \geq 1$. So, $\mathscr{C}_{\infty}$ is either empty or nonempty. $\mathscr{C}_{\infty}{ }^{F}$ is claimed by induction.
By the assumption in Iterative Scheme 1, it is observed that $F \subset \mathscr{C}=\mathscr{C}_{1}$ and $x_{1}$ is given. Now, supposing $F \subset \mathscr{C}_{m}$ for all $m \leq n$ and choosing arbitrary $z \in F$. Then, the following evaluation is obtained:

$$
\begin{aligned}
\sum_{k=1}^{m} \alpha_{n, k} \phi\left(z, T_{k} x_{n}\right) & =\sum_{k=1}^{m} \alpha_{n, k}\left(\|z\|^{2}+\left\|T_{k} x_{n}\right\|^{2}-2 \Re\left\langle z, J T_{k} x_{n}\right\rangle\right) \\
& =\sum_{k=1}^{m} \alpha_{n, k}\|z\|^{2}+\sum_{k=1}^{m} \alpha_{n, k}\left\|T_{k} x_{n}\right\|^{2}-2 \Re \sum_{k=1}^{m} \alpha_{n, k}\left\langle z, J T_{k} x_{n}\right\rangle \\
& \leq \sum_{k=1}^{m} \alpha_{n, k}\|z\|^{2}+\sum_{k=1}^{m} \alpha_{n, k}\left\|x_{n}\right\|^{2}-2 \Re \sum_{k=1}^{m} \alpha_{n, k}\left\langle z, J x_{n}\right\rangle \\
& =\|z\|^{2}+\left\|x_{n}\right\|^{2}-2 \Re\left\langle z, J x_{n}\right\rangle \\
& =\phi\left(z, x_{n}\right),
\end{aligned}
$$

where the fact that the mappings are costerro bounded linear mappings in the third step is used. Hence, it is shown that $z \in \mathscr{C}_{n+1}$. From Lemma 2, it is concluded that $\left\{\Pi_{\mathscr{C}_{n}} x\right\}_{n \geq 1}$ converges strongly to $\Pi_{\mathscr{C}_{\infty}} x$.

Lemma 7. The sequence $\left\{x_{n}\right\}_{n \geq 1}$ satisfies the inequality

$$
\left(x_{n}, \Pi_{\mathscr{C}_{n}} u\right) \leq \varepsilon_{n}^{2}
$$

Proof. Since $\Lambda\left(u, \mathscr{C}_{n}\right):=\inf _{x \in \mathscr{C}_{n}} \phi(x, u)=\phi\left(\Pi_{\mathscr{C}_{n}} u, u\right)$, then for every $\varepsilon_{n}>0, x_{n} \in \mathscr{C}_{n}$ is found such that

$$
\phi\left(x_{n}, u\right) \leq \Lambda\left(\mathscr{C}_{n}, u\right)+\varepsilon_{n}^{2}=\phi\left(\Pi_{\mathscr{C}_{n}} u, u\right)+\varepsilon_{n}^{2},
$$

which implies that

$$
\phi\left(x_{n}, u\right)-\phi\left(\Pi_{\mathscr{C}_{n}} u, u\right) \leq \varepsilon_{n}^{2} .
$$

However, Proposition 1 implies that

$$
\phi\left(x_{n}, \Pi_{\mathscr{C}} u\right) \leq \phi\left(x_{n}, u\right)-\phi\left(\Pi_{\mathscr{C}} u, u\right),
$$

and so in addition to equation (37), the following inequality is obtained:

$$
\phi\left(x_{n}, \Pi_{\mathscr{C}_{n}} u\right) \leq \varepsilon_{n}^{2},
$$

which completes the proof.

The main result of this paper is given by the following theorem.

Theorem 3 (main result). Let $\mathscr{X}$ be a uniformly convex smooth Banach space and suppose any of the following cases hold:

(i) The space $\mathscr{X}$ is finite dimensional.

(ii) The convex set $\mathscr{C}$ is compact.

(iii) $\varepsilon_{0}=0$.

Then, $\omega\left(\left\{x_{n}\right\}_{n \geq 1}\right) \neq \varnothing$ and $\omega\left(\left\{x_{n}\right\}_{n \geq 1}\right) \subseteq \cap{ }_{k=1}^{m} F\left(T_{k}\right)$, where $\omega$ denotes the (strong) limit set of the iterative sequence $\left\{x_{n}\right\}_{n \geq 1}$.
Proof. First observe that $\left\{x_{n}\right\}_{n \geq 1}$ is a bounded sequence. As a matter of fact, by Lemma 7 ,

$$
\phi\left(x_{n}, \Pi_{\mathscr{C}_{n}} u\right)=\left\|x_{n}\right\|^{2}-2 \Re\left\langle x_{n}, J \Pi_{\mathscr{C}_{n}} u\right\rangle+\left\|\Pi_{\mathscr{C}_{n}} u\right\|^{2} \leq \varepsilon_{n}^{2},
$$

which simplifies to

$$
\left\|x_{n}\right\|^{2}+\left\|\Pi_{\mathscr{C}_{n}} u\right\|^{2}-2\left\|x_{n}\right\|\left\|\Pi_{\mathscr{C}_{n}} u\right\|=\left(\left\|x_{n}\right\|-\left\|\Pi_{\mathscr{C}_{n}} u\right\|\right)^{2} \leq \varepsilon_{n}^{2} .
$$

Hence, $\left\|x_{n}\right\| \leq\left\|\Pi_{\mathscr{C}_{n}} u\right\|+\varepsilon_{n}$. So by Lemma 6 and the conditions in Iterative Scheme 1 , it is obtained $\left\{x_{n}\right\}_{n \geq 1}$ is a bounded sequence.

Consider the following cases stated in Theorem 3.

Case (i): given that $\left\{x_{n}\right\}_{n \geq 1}$ is bounded and $\mathscr{X}$ is finite dimensional, then by the Bolzano-Weierstrass theorem, $\left\{x_{n}\right\}_{n \geq 1}$ has a limit point, say $z$, and by Corollary 3 , $z \in F$ and as a result, a subsequence of $\left\{x_{n}\right\}_{n \geq 1}$ converges strongly to $z \in F$.

Case (ii): given that $\mathscr{C}$ is compact, since in metric spaces, compactness implies sequential compactness, then $\left\{x_{n}\right\}_{n \geq 1}$ being a bounded sequence has a limit point, say $z$, and by Corollary $3, z \in F$ and as a result, a subsequence of $\left\{x_{n}\right\}_{n \geq 1}$ converges strongly to $z \in F$.

Case (iii): given that $\varepsilon_{0}=0$, then by Lemma 7 ,

$$
\lim _{n \longrightarrow \infty}\left\|x_{n}-\Pi_{\mathscr{C}_{n}} u\right\|=0 \text {. }
$$

By continuity of the norm function and Lemma 6 , it is obtained that $\left\{x_{n}\right\}_{n \geq 1} \longrightarrow \Pi_{\mathscr{C}_{\infty}} u$ and as result $\Pi_{\mathscr{C}_{\infty}} u \in F$ since it is the only limit point of the sequence $\left\{x_{n}\right\}_{n \geq 1}^{\infty}$.

Remark 2. We also note that for infinite dimensions, we can also say that the sequence $\left\{x_{n}\right\}_{n \geq 1}$ has a weak limit point since a uniformly convex smooth Banach is a reflexive space. 


\section{Data Availability}

No data were used as far as this research is concerned.

\section{Conflicts of Interest}

The author declares that there are no conflicts of interest.

\section{Acknowledgments}

The author thanks colleagues for their proof reading.

\section{References}

[1] E. Picard, "Mémoire sur la théorie des équations aux dérivées partielles et la méthode des approximations successives," Journal de Mathématiques pures et appliquées, vol. 6, pp. 145-210, 1890.

[2] E. Lindelöf, "Sur l'application de la méthode des approximations successives aux équations différentielles ordinaires du premier ordre," Comptes rendus hebdomadaires des séances de l'Académie des sciences, vol. 116, no. 3, pp. 454-457, 1894.

[3] J. F. Tang and S. S. Chang, "Strong convergence theorem for a generalized mixed equilibrium problem and fixed point problem for a family of infinitely nonexpansive mappings in hilbert spaces," Pan-American Mathematical Journal, vol. 19, no. 2, pp. 75-86, 2009.

[4] M. V. Solodov and B. F. Svaiter, "Forcing strong convergence of proximal point iterations in a hilbert space," Mathematical Programming, vol. 87, no. 1, pp. 189-202, 2000.

[5] W. Takahashi, Convex Analysis and Approximation of Fixed Points, Yokohama Publishers, Yokohama, Japan, 2000.

[6] W. Takahashi, Introduction to Nonlinear and Convex Analysis, Yokohama Publishers, Yokohama, Japan, 2009.

[7] E. Blum and W. Oettli, "From optimization and variational inequalities to equilibrium problems," Mathematics Student, vol. 63, pp. 123-145, 1994.

[8] B. Halpern, "Fixed points of nonexpanding maps," Bulletin of the American Mathematical Society, vol. 73, no. 6, pp. 957962, 1967.

[9] W. R. Mann, "Mean value methods in iteration," Proceedings of the American Mathematical Society, vol. 4, no. 3, p. 506, 1953.

[10] S. Ishikawa, "Fixed points by a new iteration method," Proceedings of the American Mathematical Society, vol. 44, no. 1, p. 147, 1974.

[11] Y. Haugazeau, "Sur les inéquations variationnelles et la minimisation de fonctionnelles convexes," Thesis, Universite de Paris, Paris, France, 1968.

[12] W. Takahashi, Y. Takeuchi, and R. Kubota, "Strong convergence theorems by hybrid methods for families of nonexpansive mappings in hilbert spaces," Journal of Mathematical Analysis and Applications, vol. 341, no. 1, pp. 276-286, 2008.

[13] K. Yasunori, "Approximation of a common fixed point of a finite family of nonexpansive mappings with nonsummable errors in a hilbert space," Journal of Nonlinear and Convex Analysis, vol. 15, pp. 429-436, 2014.

[14] J. Ezearn and E. Prempeh, "A note on a shrinking projection method with nonsummable errors in a hilbert space due to Y. Kimura," Journal of Nonlinear and Convex Analysis, vol. 19, no. 14, pp. 663-667, 2018.

[15] G. Lumer, "Semi-inner-product spaces," Transactions of the American Mathematical Society, vol. 100, no. 1, p. 29, 1961.
[16] Ya I. Alber, "Generalized projection operators in banach spaces: properties and applications," 1993, https://arxiv.org/ abs/funct-an/9311002.

[17] J. Li, "The generalized projection operator on reflexive banach spaces and its applications," Journal of Mathematical Analysis and Applications, vol. 306, no. 1, pp. 55-71, 2005.

[18] J. Ezearn, Fixed point theory of some generalisations of lipschitz mappings with applications to linear and non-linear problems, $\mathrm{PhD}$. Thesis, Kwame Nkrumah University of Science and Technology, Kumasi, Ghana, 2017.

[19] Y. I. Alber, "Metric and generalized projection operators in banach spaces: properties and applications," Lecture Notes in Pure and Applied Mathematics, pp. 15-50, Marcel Dekker, New York, NY, USA, 1996.

[20] Y. I. Alber and S. Reich, "An iterative method for solving a class of nonlinear operator equations in banach spaces," PanAmerican Mathematical Journal, vol. 4, no. 2, pp. 39-54, 1994.

[21] S. Kamimura and W. Takahashi, "Strong convergence of a proximal-type algorithm in a banach space," SIAM Journal on Optimization, vol. 13, no. 3, pp. 938-945, 2002. 